\title{
A Case of Solitary Fibrous Tumor of the Mesorectum: Usefulness of Immunohistochemical Staining for a Differential Diagnosis from a Gastrointestinal Stromal Tumor
}

\author{
Nagasawa $\mathrm{Y}^{* 1,2}$, Tatsuta $\mathrm{T}^{2}$, Mukaisho $\mathrm{KI}^{3}$, Hironaka $\mathrm{T}^{2}$, Tani $\mathrm{M}^{1}$, and Kodama $\mathrm{M}^{2}$ \\ ${ }^{1}$ Department of Surgery, Shiga University of Medical Science, Otsu, Japan \\ ${ }^{2}$ Department of Surgery, Tesseikai Neurosurgical Hospital, Shijonawate, Japan \\ ${ }^{3}$ Department of Pathology, Division of Molecular and Diagnostic Pathology, Shiga University of Medical Science, Otsu, \\ Japan
}

*Corresponding author: Nagasawa Y, Department of Surgery, Shiga University of Medical Science, Seta Tsukinowacho, Otsu, Shiga 520-2192, Japan, Tel: +81 77548 2238; E-mail: ynaga@belle.shiga-med.ac.jp

Citation: Nagasawa Y, Tatsuta T, Mukaisho KI, Hironaka T, Tani M, et al. (2017) A Case of Solitary Fibrous Tumor of the Mesorectum: Usefulness of Immunohistochemical Staining for a Differential Diagnosis from a Gastrointestinal Stromal Tumor. J Cancer Sci Clin Oncol 4(1): 103. doi: 10.15744/2394-6520.4.103

Received Date: January 03, 2017 Accepted Date: May 17, 2017 Published Date: May 21, 2017

\begin{abstract}
Extrapleural solitary fibrous tumors (SFT) and gastrointestinal stromal tumors (GISTs) are similar morphologically and on imaging studies. We report a case of a patient with a giant pelvic tumor, which was difficult to be diagnosed. A 71-year-old male presented to our hospital with a complaint of urinary retention. A radiological examination showed a giant pelvic tumor mass, which ventrally compressed the urinary bladder and rectum, and was supplied by vascular flow from the right internal iliac artery. The resected specimen was $15 \times 10 \mathrm{~cm}$, elastic soft, and solid pale yellow in color. Because the tumor was histologically composed of spindle-shaped cells and was CD34 positive, cytokeratin negative, epithelial membrane antigens negative and KIT negative, we first thought the tumor was a KIT-negative GIST. However, additional immunohistochemical staining was DOG1-negative but CD99 and bcl-2 positive. Therefore, we finally diagnosed the patient with SFT. In conclusion, we report a rare case of SFT of the mesorectum to emphasize the importance of bearing this entity in mind and to show that immunohistochemical staining is useful for differentiating this type of tumor from other spindle cell neoplasms, particularly GISTs.
\end{abstract}

List of Abbreviations: SFT: Solitary fibrous tumor; GIST: Gastrointestinal stromal tumor; CT: Computed tomography; MRI: Magnetic resonance imaging; SMA: Alpha-smooth muscle actin; CK: Cytokeratin; EMA: Epithelium membrane antigens

Keywords: Solitary fibrous tumor; Mesorectum; Gastrointestinal stromal tumor

\section{Introduction}

Solitary fibrous tumor (SFT) is a rare neoplasm arising from mesenchymal tissues. SFTs were first described in the pleura in 1931 by Klemperer and Rabin [1]. SFTs are thought to represent less than $2 \%$ of all soft tissues and a morbidity rate of 2.8 per 100,000 people [2,3]. In particular, SFT arising from the mesorectum is very rare, although sporadic cases of SFTs in various extrapleural sites, such as the face, brain, peritoneum, soft tissue, liver, thyroid gland, epicardium, nasal cavity, and retroperitoneum, have been increasingly reported [4-13]. SFTs are difficult to diagnose because of various exclusion diagnoses and the requirement for immunohistochemical staining. We experienced the case of a patient complaining of urinary retention and constipation with a giant pelvic tumor, which was difficult to be diagnosed because gastrointestinal stromal tumors (GISTs) and extrapleural SFTs are similar on some imaging modalities and in morphology. Image inspection and immunostainings are useful in differential diagnosis of soft tissue tumor.

\section{Case Presentation}

A healthy 71-year-old male visited our hospital because of urinary retention and constipation. He had given himself enemas for constipation for 1 year. He came to the emergency room of our hospital complaining of urinary retention. We carried out a urethral catheterization. Serum carcinoembryonic antigen and cancer antigen 19-9 levels were normal, and other blood tests were normal. Abdominal ultrasonography showed an internal heterogenic mass of about $12 \mathrm{~cm}$. A contrast-enhanced computed tomography (CT) scan showed a giant $15 \mathrm{~cm}$ diameter intrapelvic tumor, which ventrally compress the urinary bladder and rectum but not invade into surrounding tissues (Figure 1) and reveals that the tumor was supplied by vascular 
flow from the right internal iliac artery (Figure 2). Subsequently, magnetic resonance imaging (MRI) revealed the tumor as low intensity on a T1 weighted image (Figure 3a) and high intensity on a T2 weighted image with internal homogeneity (Figure 3b). Internal homogeneity in MRI images suggested that this tumor is not sarcoma including synovial sarcoma or Ewing sarcoma family of tumors. High intensity on a T2 weighed image could exclude desmoid tumor. We suspected GIST by these findings of MRI and CT. A colonoscopy revealed extrinsic compression but no findings of intraluminal exposure of the tumor. We did not perform endoscopic biopsy, because we had been afraid of rectal perforation or penetration. Based on these findings, the lesion was suggestive of a giant soft tissue pelvic tumor, including GIST. Before surgery, we had presumed the giant tumor without findings of invasion into surrounding tissues would be located in the mesorectum, so we planned the enucleation. In surgery, the blood supply to this tumor was very rich, and the feeding arteries originated from the right internal artery, which was ligated to lessen the blood loss. The rectal wall and tumor were separated and the tumor was resected without injuring the rectum. We injected indocyanine green from the anus and confirmed no rectal injury. The macroscopic findings revealed a mass measuring $15 \times 10 \mathrm{~cm}$ that was elastic, soft, and solid pale yellow in color. The pathological examination revealed that the tumor was composed of patternless spindle-shaped cells under hematoxylin-eosin staining (Figure 4) and showed one mitotic figure/10 high-power field. Immunohistochemical staining was performed, and details of the first antibody and immunoantigen pretreatment are shown in Table I. The tumor was negative for KIT, alpha-smooth muscle actin (SMA), and S-100, but positive for CD34 (Figure 5). Furthermore, Cytokeratin (CK) and epithelium membrane antigens (EMA) was negative (Figure 6a, 6b). We initially thought the tumor was a KIT-negative GIST due to positivity for CD34. However, a mesorectal GIST is very rare, so we performed additional immunohistochemical staining to confirm the diagnosis. The additional staining was negative for DOG1, but positive for CD99 and bcl-2 (Figure 7a-7c). The Ki-67 labeling index was $<1 \%$ (Figure 7d). Thereby, we finally diagnosed a mesorectal SFT with a high risk of recurrence and metastasis. The postsurgical course was uneventful, and the patient is alive 24 months after the operation.

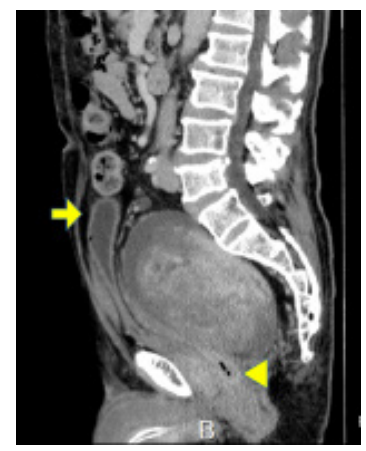

Figure 1: Contrast-enhanced computed tomography scan shows a giant tumor occupying the pelvic space and compressing the bladder (arrow) and rectum (arrowhead).

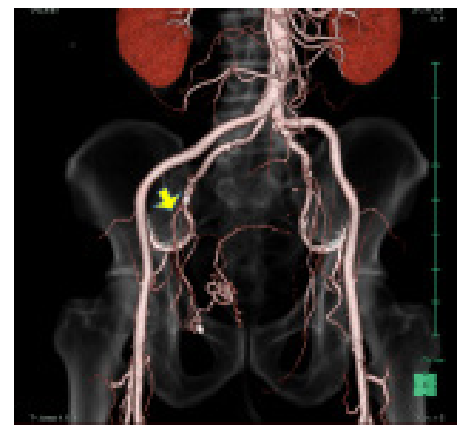

Figure 2: Contrast-enhanced computed tomography reveals that feeding artery (arrow) into the tumor originated from the right internal iliac artery.

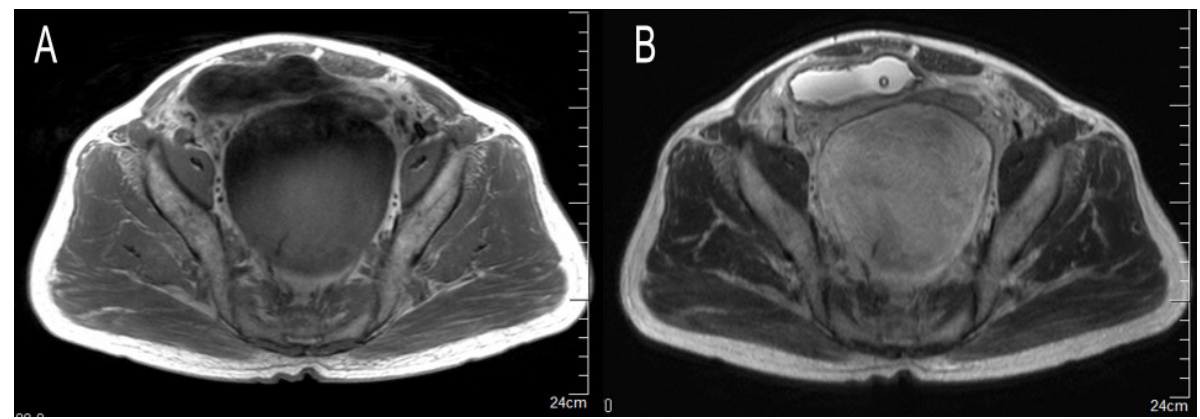

Figure 3: Magnetic resonance imaging reveals intrapelvic tumor, which shows low intensity on T1 weighted image (a) high intensity on T2 weighted image and internal homogeneity (b). 


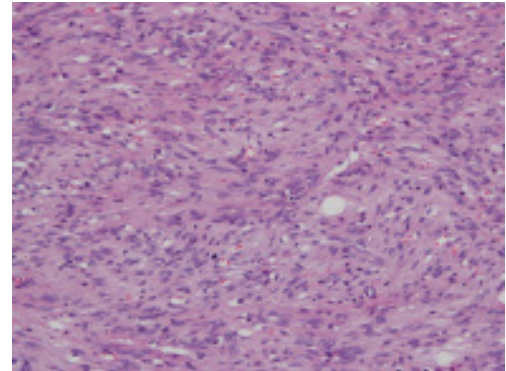

Figure 4: Hematoxylin-eosin staining shows patternless spindle-shaped cells. (HEx200)

\begin{tabular}{|c|c|c|c|c|c|}
\hline Antigen & Antibody & Antibody maker & Machinery & Pretreatment & $\begin{array}{l}\text { Primary antibody } \\
\text { condition }\end{array}$ \\
\hline Kit & 9.7 & Nippon Roche, Tokyo & Ventana Benchmark XT & $\mathrm{pH} 8.5,60 \mathrm{~min}$ & $37^{\circ} \mathrm{C}, 32 \mathrm{~min}$ \\
\hline CD34 & QBEnd/10 & Nippon Roche, Tokyo & Ventana Benchmark XT & $\mathrm{pH} 8.5,60 \mathrm{~min}$ & $37^{\circ} \mathrm{C}, 32 \mathrm{~min}$ \\
\hline S-100 & polyclonal & Nippon Roche, Tokyo & Ventana Benchmark XT & none & $37^{\circ} \mathrm{C}, 16 \mathrm{~min}$ \\
\hline SMA & $1 \mathrm{~A} 4$ & Nippon Roche, Tokyo & Ventana Benchmark XT & $\mathrm{pH} 8.5,60 \mathrm{~min}$ & $37^{\circ} \mathrm{C}, 32 \mathrm{~min}$ \\
\hline Cytokeratin & AE1/AE3 & DAKO, Tokyo & Ventrana Benchmark XT & Peroxidase $4 \mathrm{~min}$ & $37^{\circ} \mathrm{C}, 32 \mathrm{~min}$ \\
\hline EMA & E29 & DAKO, Carpinteria & Ventrana Benchmark XT & $\mathrm{pH} 8.5,60 \mathrm{~min}$ & $37^{\circ} \mathrm{C}, 32 \mathrm{~min}$ \\
\hline DOG-1 & K9 & Leica, Tokyo & Leica BOND III & $\mathrm{pH} 9.0,20 \mathrm{~min}$ & $\begin{array}{c}\text { room tempurature, } \\
15 \mathrm{~min}\end{array}$ \\
\hline CD99 & $12 \mathrm{E} 7$ & DAKO, Tokyo & Leica BOND III & $\mathrm{pH} 9.0,20 \mathrm{~min}$ & $\begin{array}{l}\text { room tempurature, } \\
15 \mathrm{~min}\end{array}$ \\
\hline bcl-2 & 124 & DAKO, Tokyo & Leica BOND III & $\mathrm{pH} 9.0,20 \mathrm{~min}$ & $\begin{array}{c}\text { room tempurature, } \\
15 \mathrm{~min}\end{array}$ \\
\hline Ki-67 & $30-9$ & Nippon Roche, Tokyo & Ventana Benchmark XT & $\mathrm{pH} 8.5,60 \mathrm{~min}$ & $37^{\circ} \mathrm{C}, 16 \mathrm{~min}$ \\
\hline
\end{tabular}

Table 1: Details of antibody and immunoantigen pretreatment

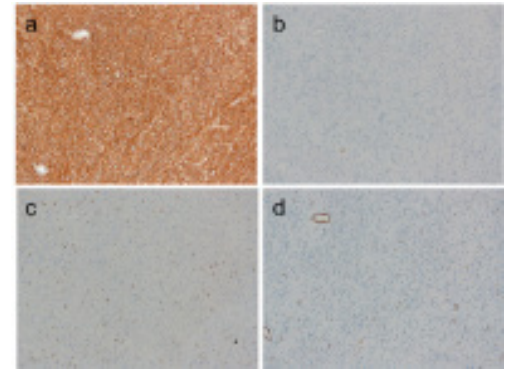

Figure 5: Surgical specimen was positive for CD34 (a) and negative for S-100 (b), KIT(c) and alpha-smooth muscle actin (SMA) (d). (x100)

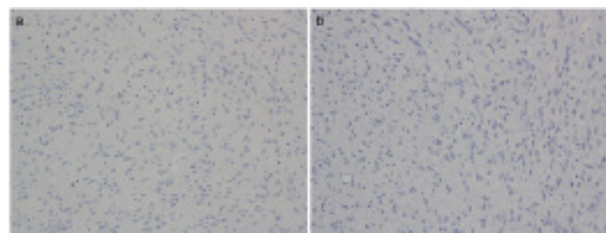

Figure 6: Surgical specimen was negative for cytokeratin (CK) (a) and for endothelial membrane antigen (EMA) (b).(x100)

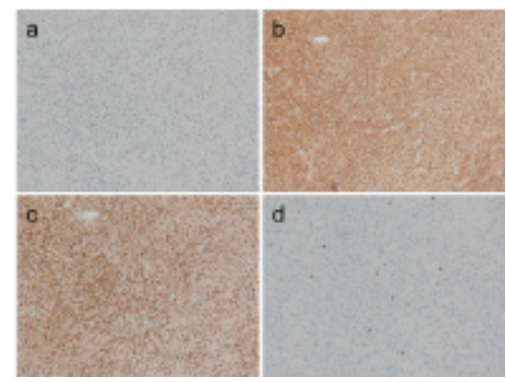

Figure 7: Specimen is negative for DOG1 (a) and positive for CD99 (b) and bcl-2 (c). Ki-67 index was $<1 \%$ (d). (x100) 


\section{Discussion}

We report a rare case of SFT of the mesorectum to emphasize the importance of bearing this entity in mind and to show that immunohistochemical staining including DOG1 is useful for differentiating a SFT from a GIST.

Because GISTs and extrapleural SFTs are similar on some imaging modalities and in morphology, SFTs and GISTs are difficult to diagnose [14]. Imaging shows various and nonspecific findings [15]. Because SFTs can occur in many locations with varied tumor morphology, differentiating a SFT from other similar tumors including GISTs requires cytomorphology and immunohistochemical staining studies $[16,17]$. However, it is often difficult to distinguish a SFT from a GIST despite immunohistochemical staining. A considerable number of studies have shown that hematoxylin-eosin stained spindle-shaped tumors require immunohistochemical support, such as KIT, CD34, alpha-SMA, and S-100 proteins, to differentiate a GIST from other soft tissue tumors, including SFTs. According to Vinod, the major difference between a GIST and a SFT is KIT [18]. The present case was negative for KIT, alpha-SMA, and S-100, but positive for CD34. We first considered that the tumor was a GIST because the frequency of KIT-negative GISTs is about 30\% [19]. However, a GIST arising from the mesorectum is very rare. So, we added additional immunohistochemical staining antibodies for the differential diagnosis. Some reports have revealed that DOG1 is useful to diagnose a GIST. Nazneen reported that the DOG1 antibody has 100\% sensitivity and $100 \%$ specificity to diagnose a GIST and that all KIT negative GISTs are positive for DOG1 [19]. The differential diagnosis from GIST to SFT required CD99, bcl-2, and DOG1 as additional immunohistochemical staining in the present study. Most SFTs are CD99 positive and bcl-2 positive [20,21].

It has been reported that $10-25 \%$ of SFTs recur. A risk stratification model for a SFT is composed of age, tumor size, and mitotic figures. In our case, the patient was 71 years old $(>55)$, tumor size was $15 \mathrm{~cm}$, and the tumor had one mitotic figure per high-power field, so he fell in the high risk group. SFTs can recur for up to 24 years [22]. SFTs mainly spread through the blood stream and the most common metastatic sites are the lungs, liver, and bone [22]. According to Bouhabel, tumors $>50$ $\mathrm{mm}$, with dense cellularity, pleomorphism in the nucleus, mitotic activity ( $>4$ in 10 high-power fields), anaplasia, necrosis, and hemorrhage are mostly malignant [22]. Malignant SFTs occur in 20\% of cases but malignancy does not predict the prognosis [23]. The 5-year survival rate of a patient with a tumor $>10 \mathrm{~cm}$ is only $20 \%$ [24].

The best treatment for a SFT is surgical en bloc resection of the mass. Adjuvant chemotherapy or systemic chemotherapy can be used for a GIST. Small case series and retrospective studies have reported using anthracyclines plus ifosfamide, dacarbazine, or a combination of bevacizumab and temozolomide [25-27]. Intrapelvic SFTs often rich blood flow from the iliac artery or inferior mesenteric artery so resection of the tumor results in severe bleeding [4,28]. In the present case, we ligated the feeding artery from the right internal artery in the early stage of surgery, so we reduced the amount of bleeding of the tumor.

\section{Conclusion}

The possibility of a SFT should be considered when a tumor is diagnosed as a KIT-negative GIST due to positivity for CD34. Additional immunohistochemical staining with CD99, bcl-2, and DOG1 may be useful for the differential diagnosis.

\section{References}

1. Paul Klemperer and Coleman B. Rabin (1992) Primary neoplasm of the pleura: a report of five cases. Am J Ind Med 22: 1-31.

2. Gold JS1, Antonescu CR, Hajdu C, Ferrone CR, Hussain M et al. (2002) Clinicopathologic Correlates of Solitary Fibrous Tumors. Cancer 94: 1057-68.

3. Chick JFB, Chauhan NR, Madan R (2013) Solitary fibrous tumors of the thorax: Nomenclature, epidemiology, radiologic and pathologic findings, differential diagnoses, and management. AJR Am J Roentgenol 200: W238-48.

4. Soda H, Kainuma O, Yamamoto H, Nagata M, Takiguchi N et al. (2010) Giant intrapelvic solitary fibrous tumor arising from mesorectum. Clin J Gastroenterol 3: 136-9.

5. Venara A, Lermite E, Thibaudeau E, Ridereau-Zins C, Casa C et al. (2011) Solitary fibrous tumour of the mesorectum: A case report. Color Dis 13: 158-9.

6. Kawamura J, Tani M, Kida Y, Sumida K, Ogawa R, et al. (2016) Successful laparoscopic treatment of a giant solitary fibrous tumor of the mesorectum: A case report and literature review. Asian J Endosc Surg 10: 51-4

7. Demicco EG, Park MS, Araujo DM, Fox PS, Bassett RL et al. (2012) Solitary fibrous tumor: a clinicopathological study of 110 cases and proposed risk assessment model. Mod Pathol 25: 1298-306.

8. Satomi T, Hasegawa O, Abukawa H, Kohno M, Enomoto A et al. (2014) Exceptionally large solitary fibrous tumor arising from the cheek: An immunohistochemical and ultrastructural study with a review of the literature. Med Mol Morphol 47: 108-16.

9. Farrag TY, Micchelli S, Tufano RP (2009) Solitary fibrous tumor of the thyroid gland. Laryngoscope 119: 2306-8.

10. Tsuburaya A, Noguchi Y, Yoshikawa T, Nomura K, Fukuzawa K et al. (1993) Long-term effect of radical gastrectomy on nutrition and immunity. Surg Today 23: $320-4$.

11. Mathew GA, Ashish G, Tyagi AK, Chandrashekharan R, Paul RR (2015) Solitary Fibrous Tumor of Nasal Cavity : A Case Report. Iran J Otorhinolaryngol 27: 307-12.

12. Bianchi G, Ferrarini M, Matteucci M, Monteleone A, Aquaro GD et al. (2013) Giant solitary fibrous tumor of the epicardium causing reversible heart failure. Ann Thorac Surg 96: e49-51.

13. Maki T, Fujino S, Misu K, Kaneko H, Inomata H et al. (2016) Integrally calcified solitary fibrous tumor in the retroperitoneum: a case report and review of the literature. Surg Case Rep 2: 14. 
14. Zhang GJ, Li RT, Zhou Y, Huang F, Zhao ZC et al. (2015) Case Report Solitary fibrous tumor of small bowel mesentery with postoperative bowel obstruction : a case report and review of literature. 8 11691-7.

15. Keraliya AR, Tirumani SH, Shinagare AB, Zaheer A, Ramaiya NH (2016) Solitary Fibrous Tumors: 2016 Imaging Update. Radiol Clin North Am 54: 565-79.

16. Cristi E, Perrone G, Battista C, Benedetti-Panici P, Rabitti C (2005) A rare case of solitary fibrous tumour of the pre-sacral space: Morphological and immunohistochemical features. In Vivo 19: 777-80.

17. Balaji R, Ramachandran K, Somanathan T (2009) A rare case of solitary fibrous tumour of the sigmoid mesocolon: Imaging features and review of literature. Cancer Imaging 9: 67-9.

18. Shidham VB, Chivukula M, Gupta D, Rao RN, Komorowski R (2002) Immunohistochemical comparison of gastrointestinal stromal tumor and solitary fibrous tumor. Arch Pathol Lab Med 126: 1189-92.

19. Fatima N, Cohen C, Siddiqui MT (2011) DOG1 utility in diagnosing gastrointestinal stromal tumors on fine-needle aspiration. Cancer Cytopathol 119: 202-8. 20. Alawi F, Stratton D, Freedman PD (2001) Solitary Fibrous Tumor of the Oral Soft Tissues. A Clinicopathologic and Immunohistochemical Study of 16 Cases. Am J Surg Pathol 25: 900-10.

21. Hasegawa T, Matsuno Y, Shimoda T, Hasegawa F, Sano T et al. (1999) Extrathoracic solitary fibrous tumors: their histological variability and potentially aggressive behavior. Hum Pathol 30: 1464-73.

22. Bouhabel S, Leblanc G, Ferreira J, Leclerc YE, Dubé P et al. (2011) Solitary fibrous tumor arising in the mesentery: a case report. World J Surg Oncol 9: 140.

23. Dalton WT, Zolliker AS, McCaughey WTE, Jacques J, Kannerstein M (1979) Localized primary tumors of the pleura. An analysis of 40 cases. Cancer $44: 1465$ 75.

24. DeMatteo RP, Lewis JJ, Leung D, Mudan SS, Woodruff JM et al. (2000) Two Hundred Gastrointestinal Stromal Tumors: recurrence patterns and prognostic factors for survival. Ann Surg 231: 51-8.

25. Stacchiotti S, Libertini M, Negri T, Palassini E, Gronchi A, et al. (2013) Response to chemotherapy of solitary fibrous tumour: A retrospective study. Eur J Cancer 49: 2376-83.

26. Stacchiotti S, Tortoreto M, Bozzi F, Tamborini E, Morosi C et al. (2013) Dacarbazine in solitary fibrous tumor: a case series analysis and preclinical evidence vis-a-vis temozolomide and antiangiogenics. Clin Cancer Res 19: 5192-201.

27. Park MS, Patel SR, Ludwig JA, Trent JC, Conrad CA et al. (2011) Activity of temozolomide and bevacizumab in the treatment of locally advanced, recurrent, and metastatic hemangiopericytoma and malignant solitary fibrous tumor. Cancer 117: 4939-47.

28. Wat SY, Sur M, Dhamanaskar K (2008) Solitary fibrous tumor (SFT) of the pelvis. Clin Imaging 32: 152-6.

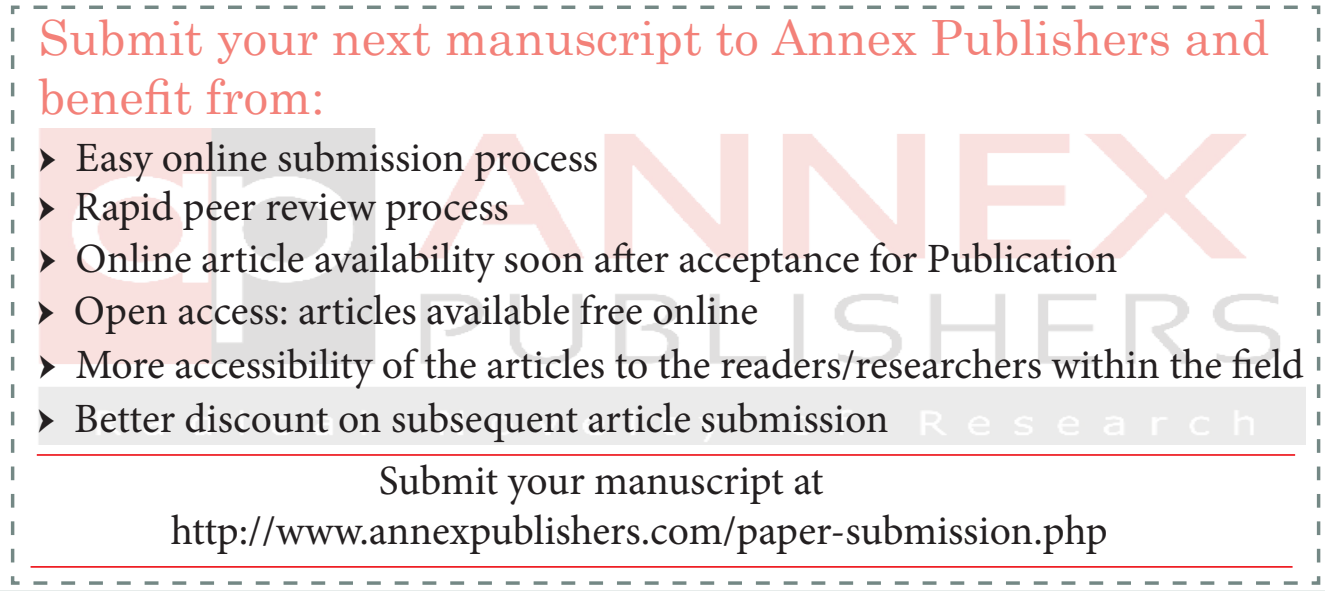

